\title{
A substantial caries-inhibiting effect of fluoride varnish is suggested
}

Marinho VCC, Higgins JPT, Logan S, Sheiham A. Fluoride varnishes for preventing dental caries in children and adolescents (Cochrane Review). The Cochrane Library 2002; Issue 3. Oxford: Update Software

Question: In children under 16 is fluoride varnish effective in preventing caries?

Objective To determine the effectiveness and safety of fluoride varnishes in the prevention of dental caries in children and to examine factors potentially modifying their effect.

Data sources The reviewers attempted to identify all relevant studies irrespective of language, from 1965 onwards. Multiple electronic database were searched together with reference lists of articles, prospective hand searches of the journals identified as publishing the most eligible trials, and searches for selected authors and manufacturers.

Study selection Randomised or quasi-randomised controlled trials were included if they had blind outcome assessment and compared fluoride varnish with placebo or no treatment in children up to 16 years old for at least 1 year. The main outcome was caries increment measured by the change in decayed, missing and filled tooth surfaces (DMFS).

Data extraction and synthesis Inclusion decisions, quality assessment and data extraction were duplicated in a random sample of one third of studies, and consensus was achieved by discussion with a third party. Study authors were contacted for missing data. The primary measure of effect was the prevented fraction $(\mathrm{PF})$, that is, the difference in caries increments between the treatment and control groups expressed as a proportion of the increment in the control group. Random effects meta-analyses were performed where data could be pooled. Potential sources of heterogeneity were examined in random-effects meta-regression analyses.

Results Nine studies were included, involving 2709 children. Seven contributed data for the main meta-analysis. There was substantial heterogeneity. The estimated pooled prevented fraction for permanent teeth was $46 \%$ (95\% CI 30-63) and 33\% (95\% CI 19-48) for deciduous teeth. No significant association between estimates of dfs, DMFS, PF and baseline caries severity or background exposure to fluorides was found in meta-regression, and a funnel plot of the seven studies indicated no relationship between PF and study precision. In both methods, power is limited when only a few trials are included.

Table 1 Numbers needed to treat (NNT)

\begin{tabular}{lccr}
\hline & \multicolumn{2}{c}{ Caries increment } & \\
\cline { 2 - 3 } & $\mathrm{dfs} /$ year & DMFS/year & NNT $(95 \% \mathrm{Cl})$ \\
\hline Deciduous teeth & & & \\
& 0.82 & & $3.7(2.5-6.4)$ \\
Permanent teeth & 1.90 & & $1.6(1.1-2.8)$ \\
& & 0.67 & $3.2(2.4-5.0)$ \\
& & 1.60 & $1.4(1.0-2.0)$ \\
\hline
\end{tabular}

$\mathrm{Cl}$, confidence interval.

Conclusions The review suggests a substantial caries-inhibiting effect of fluoride varnish in both the permanent and the deciduous dentitions based largely on trials that had no treatment controls. There is little information concerning acceptability of treatment or possible side-effects in the trials included. Given the relatively poor quality of most of the studies and the wide confidence intervals around the estimates of effect, there remains a need for further trials of high quality that include assessment of potential adverse effects.

Evidence-Based Dentistry (2002) 3, 105-106. doi:10.1038/ sj.ebd.6400142

Address for correspondence: Emma Tavender, Cochrane Oral Health Group, MANDEC, University Dental Hospital of Manchester, Higher Cambridge Street, Manchester M15 6FH, UK.

E-mail: emma.tavender@man.ac.uk

\section{Commentary}

Fluoride varnishes have been used for prevention of dental caries for more than 3 decades, and over this time a considerable literature supporting their effectiveness has appeared. Three previous meta-analyses have concluded that there is evidence for the effectiveness of fluoride varnishes in preventing dental caries. ${ }^{1-3}$ This recently published Cochrane review reaches a similar conclusion, although it was unable to satisfactorily achieve its other objectives, ie, determining the safety of fluoride varnishes and examining whether the preventive effects of fluoride varnishes are influenced by the level of caries severity or background exposure to fluoride.

The review has been thoroughly and carefully performed. Three principal exclusion criteria served to eliminate the majority of 42 identified studies of effectiveness of fluoride gels. These criteria were split mouth, or withinsubject control designs; absence of randomised or quasi-randomised allocation to treatment; and absence of blind outcome assessment. Seven studies contributed data for the effectiveness estimate for permanent teeth, and three for primary teeth. Both effectiveness estimates were positive and statis- 
tically significant, echoing the findings of two of the previous reviews for permanent teeth. ${ }^{1,2}$ The conclusion that fluoride varnish is effective for caries prevention in primary teeth is unique: such a conclusion has not been advanced previously. The review conveys these findings tentatively, stressing the poor quality of most of the included studies, the small number of these studies, and the wide confidence intervals around the effect estimates.

The inability of the review to completely achieve its other objectives is disappointing because safety issues and the differential effectiveness of varnishes - given differences in caries incidence rates and fluoride exposures - should be important determinants in both programme decisions and individual clinical decisions regarding their use. The review states that for permanent teeth, no significant relationship was found between the PF and either baseline caries level or fluoridation exposure, but pointed out also that the power to detect such effects was low. It is equally disappointing to realise that, of the 10 studies of fluoride varnish in children and adolescents published since 1990 identified in this review, six had to be excluded because of problems with randomisation or assessment blinding. With the increasing interest in evidence-based dentistry over the past decade, one would have hoped that more attention had been given to controlling threats to the internal validity of the clinical trials that comprise that evidence.

This Cochrane review of the effectiveness of fluoride varnish for the prevention of dental caries in children and adolescents is one of a series of systematic reviews of topical fluoride interventions performed by the same group of researchers. The first in the series focused on the effectiveness of fluoride gels. ${ }^{4}$ It reported a pooled PF on permanent tooth surfaces of $28 \%$ based on a total of 23 studies, and $21 \%$ based on 14 placebo-controlled studies. A significant difference in effect-estimates by type of control group was not seen in the varnish studies, although the authors decided not to present the results of that analysis for undisclosed reasons. It would seem important to do so, if only to invigorate the discussion of the influence of study methods on effect estimates.

The appearance of this Cochrane review raises an issue regarding dissemination of results. This is a technical report, and both its structure and prose may be rather impenetrable to the practicing clinician. This may not be a problem, however, since the probability of a clinician encountering one of these reports is remote; their distribution is restricted by subscription. To date, no paper describing either this review or the first in the series has appeared in the periodic literature. Thus, it is likely that clinicians either will not hear of the review or will see only a brief summary of the results. Such a condensation will not convey essential information such as the small number of studies included, the review's tentativeness in presenting the effect estimates, or the concern expressed in the review about the absence of data with which to examine safety and effect modification. Recently, Cochrane reviews began to be included in MEDLINE, but a more comprehensive approach to information transfer is needed.

The same research group used essentially identical approaches to produce both the reviews of fluoride gels and varnishes. The difference in effectestimates for these two preventive methods based on all included studies ( $28 \%$ for gels and $46 \%$ for varnishes), suggests that varnishes may be superior, although the width of associated confidence intervals indicates that, if tested, this difference would not be statistically significant. The most recent of the other three meta-analyses of fluoride varnishes used a different, and in some respects still more stringent set of inclusion criteria, which resulted in the analysis of only three studies. ${ }^{3}$ All three of these studies were comparisons of fluoride varnishes with fortnightly fluoride rinses. Again, the fluoride varnish outperformed the rinse, but the results were not statistically significant. Although far from convincing, these results certainly suggest that there is a need for additional studies to determine whether fluoride varnishes are more effective in caries prevention than fluoride gels or rinses. If such an advantage is seen, a stronger case for adoption of varnish over rinses and gels exists. Varnishes are already perceived to present some empirical advantages as a preventive intervention. Arguably, varnishes lead to less fluoride ingestion, which may be of importance in young children and other vulnerable populations. ${ }^{3,5}$ They are certainly less prone to protocol modification (reduction in application time to $1 \mathrm{~min}$ ) by practitioners, a current problem in the US with unknown implications for effectiveness. ${ }^{6}$ Finally, patient acceptance is reportedly greater by adults. ${ }^{7}$

1. Clark D, Hanley J, Stamm J, Weinstein P. An empirically based system to estimate the effectiveness of caries-preventive agents. A comparison of the effectiveness estimates of APF gels and solutions, and fluoride varnishes. Caries Res 1985; 19:83-95.

2. Helfenstein U, Steiner M. Fluoride varnishes (Duraphat): a meta-analysis. Community Dent Oral Epidemiol 1994; 22:1-5.

3. Strohmenger L, Brambilla E. The use of fluoride varnishes in the prevention of dental caries: a short review. Oral Dis 2001; 7:71-80.

4. Marinho V, Higgins J, Logan S, Sheiham A. Fluoride gels for preventing dental caries in children and adolescents (Cochrane Review). In: The Cochrane Library, Issue 3 2002. Oxford: Update Software.

5. Bawden J. Fluoride varnish: a useful new tool for public health dentistry. J Public Health Dent 1998; 58:266-269.

6. Chan J, Warren D, Henson H. Use of inoffice fluorides in the Greater Houston area. J Greater Houston Dent Soc 1996; 68: 22-42.

7. Warren D, Henson H, Chan J. Dental hygienist and patient comparisons of fluoride varnishes to fluoride gels. J Dent Hyg 2000; 74:94-101.

Jim Bader Department of Operative Dentistry, University of North Carolina, Chapel Hill, North Carolina, USA 